\title{
Evaluation, Refurbishment, and Characterization of Fast Neutron Scintillators for the TREAT Fuel Motion Monitoring System
}

Jay D. Hix, David L. Chichester, Scott M. Watson, James T. Johnson, Scott J. Thompson

October 2017

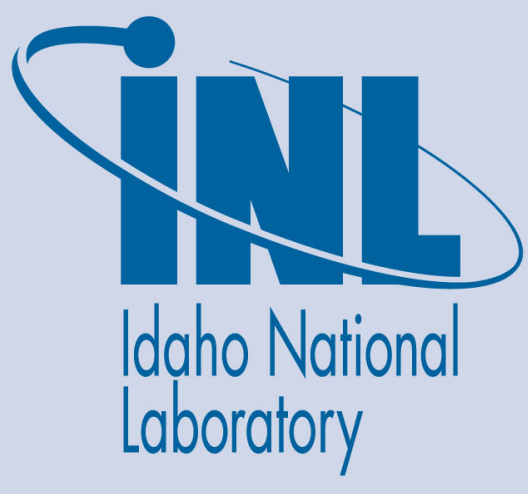

The INL is a U.S. Department of Energy National Laboratory operated by Battelle Energy Alliance 


\section{Evaluation, Refurbishment, and Characterization of Fast Neutron Scintillators for the TREAT Fuel Motion Monitoring System}

Jay D. Hix, David L. Chichester, Scott M. Watson, James T. Johnson, Scott J.

Thompson

October 2017

Idaho National Laboratory Idaho Falls, Idaho 83415

http://www.inl.gov

Prepared for the

U.S. Department of Energy

Under DOE Idaho Operations Office

Contract DE-AC07-05ID14517 


\title{
Evaluation, Refurbishment, and Characterization of Fast Neutron Scintillators for the TREAT Fuel Motion Monitoring System
}

\author{
Jay D. Hix, Student Member IEEE, David L. Chichester Sr. Member IEEE, Scott M. Watson, Member IEEE, James \\ T. Johnson, Member IEEE, and Scott J. Thompson, Member IEEE
}

\begin{abstract}
This paper details with work being performed to evaluate, refurbish, and characterize proton-recoil scintillator (PRS) assemblies being used in a 360-channel detector array for the measurement of fast neutrons in the Fuel Motion Monitoring System (FMMS) at the Transient Reactor Test Facility (TREAT). The TREAT FMMS measures the real-time movement and displacement of experimental fuel during transient experiments, correlating fast-neutron signals to the mass of fuel in each of 360 pixels at the center of the reactor's core. The detectors in the FMMS have stood dormant for over 20 years and significant degradation must be addressed before the system can be brought back to operational status. The PRS refurbishment process began by decoupling the scintillators from their matched photomultiplier tubes (PMTs) and treating the PRSs with ethanol. This process cleans the surfaces, removing paint and legacy labels/markings. During this procedure many physical variations were observed. After cleaning, the PRSs were measured using a digital caliper and the dimensions documented. Following this, a well-controlled fiber-coupled $280-\mathrm{nm}$ light-emitting diode light source was used to stimulate the PRSs to produce an emission spectrum inside a light integration sphere. This emission spectrum was measured using an ultra-violet light enhanced optical spectrometer. Spectral measurements were recorded from $185 \mathrm{~nm}$ to $580 \mathrm{~nm}$ in $0.4-\mathrm{nm}$ steps. The PRSs were then lightly sanded on the non-PMT mating surfaces and painted with commercially available reflective paint. A final emission spectrum was recorded for each PRS after painting.
\end{abstract}

\section{INTRODUCTION}

The Fuel Motion Monitoring System (FMMS) is a fast

Abstract submitted May 5, 2017. This work was supported by the U.S Department of Energy Nuclear Science User Facilities.

Jay D. Hix- Idaho National Laboratory, Idaho Falls, ID 83415 USA

(telephone: 208-526-3972, e-mail: jay.hix@inl.gov).

David L. Chichester - Idaho National Laboratory, Idaho Falls, ID 83415

USA (telephone: 208-526-8920, e-mail: david.chichester@inl.gov).

Scott M. Watson - Idaho National Laboratory, Idaho Falls, ID 83415 USA (telephone: 208-525-5403, e-mail: scott.watson@inl.gov).

James T. Johnson - Idaho National Laboratory, Idaho Falls, ID 83415 USA (telephone: 208-525-5402, e-mail: jame.johnson@inl.gov).

Scott J. Thompson - Idaho National Laboratory, Idaho Falls, ID 83415 USA (telephone: 208-526-1459, e-mail: scott.thompson@inl.gov). neutron detector system capable of measuring the motion of experimental nuclear fuel in a test capsule placed in the center of the core of the Transient Reactor Test Facility (TREAT) as the fuel fails in response to simulated, accident-like conditions. There are two collimators associated with the FMMS. The front collimator is a horizontal slit with an aperture $38.1-\mathrm{mm}$ tall by $215.6-\mathrm{mm}$ wide that provides general shielding of the rear collimator. The rear collimator is a stack of steel plates with 360 milled, angled slots. The rear collimator projects line-of-sight neutrons from the center of the reactor's core to an array of proton-recoil scintillator (PRS) fast-neutron detectors. The PRS detectors have been sitting dormant for over 20 years and substantial analysis of each PRS must be addressed before being considered a candidate for refurbishment.

\section{METHODS}

The FMMS features 360 channels that make up a 10 by 36 detector array consisting of PRS buttons matched to photomultiplier tubes (PMTs), one on each channel slot. A PRS consists of a blend of $5 \% \mathrm{ZnS}(\mathrm{Ag})$ grains dispersed in a 95\% Lucite medium to produce a wafer with a dimension of $2.78 \mathrm{~mm} \times 12.26 \mathrm{~mm} \times 15.90 \mathrm{~mm}$. The $\mathrm{ZnS}(\mathrm{Ag}) /$ Lucite wafer is then adhered to two half-cylindrical Lucite waveguides using optical cement, forming a PRS button. Each PRS button is coupled to a PMT with an optical gel, yielding a PRS detector assembly. The fast neutrons are detected from light produced by the recoil of protons interacting with the $\mathrm{ZnS}(\mathrm{Ag})$ grains.[1-3]

\section{A. Proton Recoil Scintillator Refurbishment and Selection}

After reclaiming 400 original PRS detectors from the FMMS at TREAT, the examination and cleaning process began. The PRS buttons were decoupled from their PMTs and then soaked in ethanol for an average of 40 minutes to clean the original paint and markings off the PRS wafers and waveguides. Afterwards, a cotton tip applicator was used to clean the residual paint between the scintillator and waveguides to ensure all the original paint and markings were removed. When all the original paint has been removed each PRS button was visually inspected. When inspecting the PRS buttons many physical variations were observed. Variations included scintillator discoloration (yellow and white), aligned 
and misaligned waveguide geometries, sanded and non-sanded PRS surfaces, delamination between scintillator and waveguide, and epoxy left on PRS surfaces. The majority of the PRS buttons have some degree of misalignment and delamination. The misalignment of the PRS could be due to the hand making of the buttons. The degree of delamination could be caused by the epoxy drying faster in some spots before the scintillator and the light guides were placed together presenting a delaminated aspect from the outside in. Also, over time the epoxy could have lost its strength, displaying a delaminated appearance. It was observed that only limited quantities were in proper alignment and displayed no delamination.

These physical variations between individual PRS buttons were quite evident upon visual inspection. Some physical variations were correlated to the PRS light output emission. These include scintillator discoloration (yellowing) along with sanded PRS surfaces, both of which were observed to correlate with lower PRS light emission as measured in a light-integrating sphere. Overall, scintillators that were white in color have higher emission intensities than those scintillators that are yellow. An example of the lower count rate is shown in Figure 1. The spectrogram of a white PRS button is shown in black, and a yellowed PRS button is shown in yellow; a 280-nm light-emitting diode (LED) was use for illumination. The peak count values are the maximum amplitude of the spectrograms at a wavelength of $\sim 452 \mathrm{~nm}$.

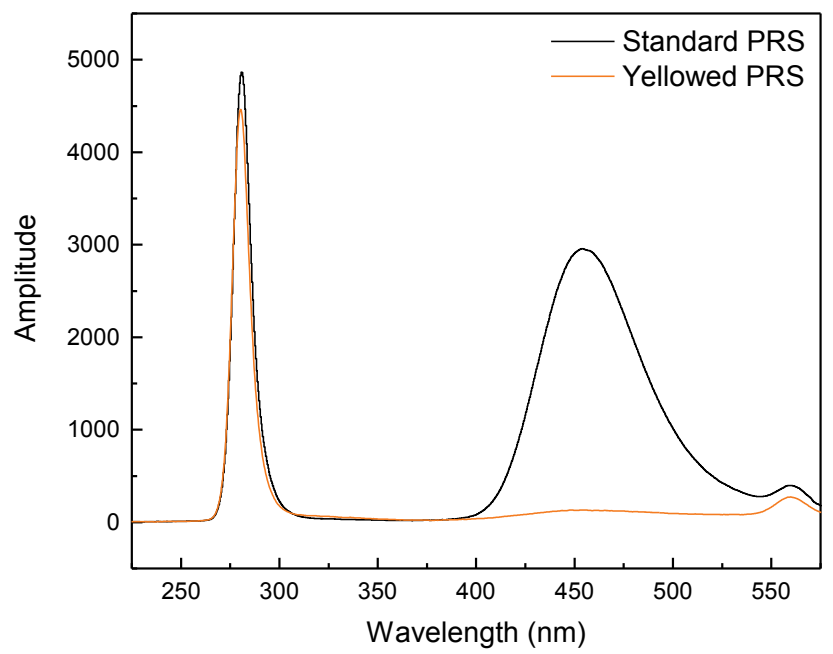

Figure 1 This plot shows a spectrogram with 280-nm stimulation light and the 452-nm output emission light from two PRS buttons under test, one with a white scintillator and the other with a yellowed scintillator. The small peak at $560 \mathrm{~nm}$ is a result of half-harmonic generation from the 280-nm LED.

The yellowing of the $\mathrm{ZnS}(\mathrm{Ag})$ wafers may be due to age, prolonged exposure to light, humidity, contact with ionizing radiation, or variations in manufacturing. When comparing two PRS buttons, one with a yellowed wafer and the other a white wafer, it is evident that the PRS button with the yellowed wafer has a greatly reduced light output emission when compared to the PRS with a bright-white wafer.

The majority of the PRS buttons have some degree of misalignment or delamination, perhaps because they were handmade. Only a hand full of observed PRS buttons had yellowing scintillators. After analyzing all of the PRS buttons, 118 refurbishment candidates were selected out of the 400 reclaimed PRS buttons. To be considered a candidate the PRS buttons must hold the following characteristics:

- $\quad$ The scintillator must be white

- It must not show any visual signs of delamination.

- The Lucite waveguides must be in alignment, and there must be no sign of scratches or other markings that could affect light emission output
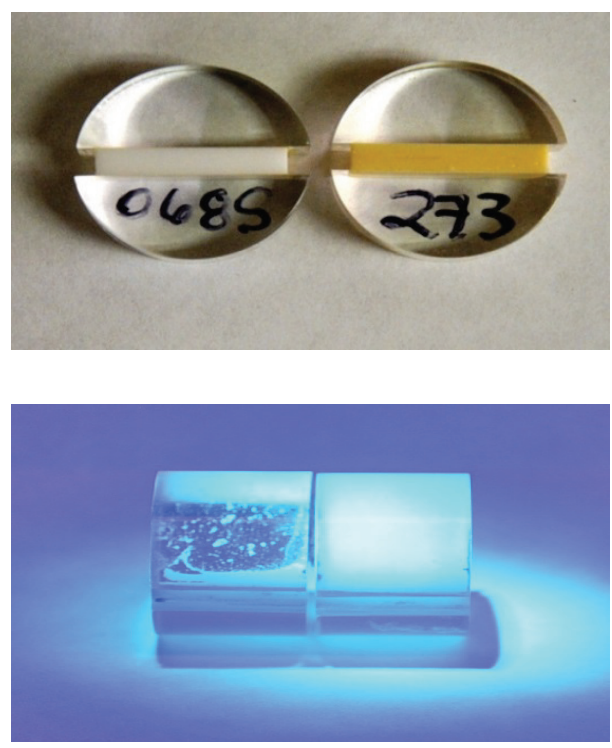

Figure 2 The top picture illustrates the difference between a white and a yellowed PRS wafer. The picture on the bottom gives an example of a PRS button that is starting to delaminate (left) between the scintillator wafer and waveguide.

\section{B. Proton Recoil Scintillator Re-Painting Process}

Once all of the PRS buttons were cleaned, measured, and sorted, the identified candidates were prepared for the next step in the process. The PRS buttons were sanded using 500grit sandpaper. The buttons were sanded on the top and the sides while the bottom of the button was left un-sanded but polished, for coupling to a PMT. After each button was sanded, and washed again using ethanol, they were ready for re-painting using Eljen Technologies EJ-510 reflecting coating. Each button was placed on a turntable and spun as paint was applied by using an air brush, to achieve even distribution of the paint. Thus process is shown in Figure 4. 

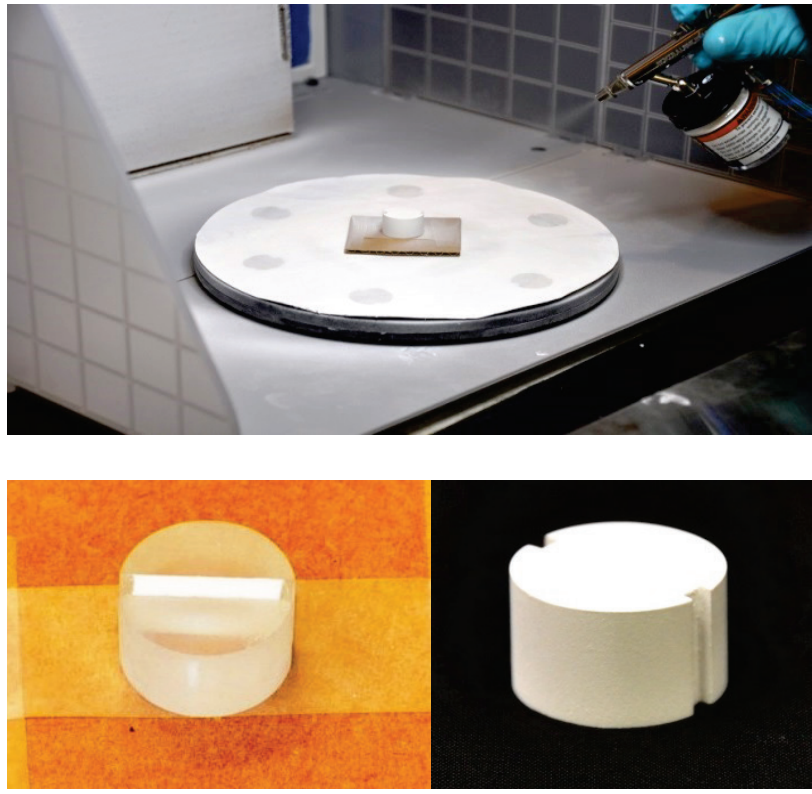

Figure 4. The top picture shows re-painting of a reclaimed PRS button. The bottom picture illustrates a before-and-after painting comparison.

\section{Reflective finish performance}

The PRS scintillators exhibit excellent neutron-photon pulse shape discrimination. A small study was performed to assess if specular (reflective) paint would perform better than diffuse (white) paint for this application. A standard pulse-shape discrimination (PSD) figure-of-merit (FOM) was used to characterize the performance of a Rust-Oleum mirror finish, EJ-510 paint, and a clear PRS. Measurements were performed for 900 seconds in each case, using a ${ }^{252} \mathrm{Cf}$ neutron source for irradiation. The data collected in each case are shown in Figure 5 and are tabulated in Table I. The button with EJ-510 paint showed the best PSD FOM.

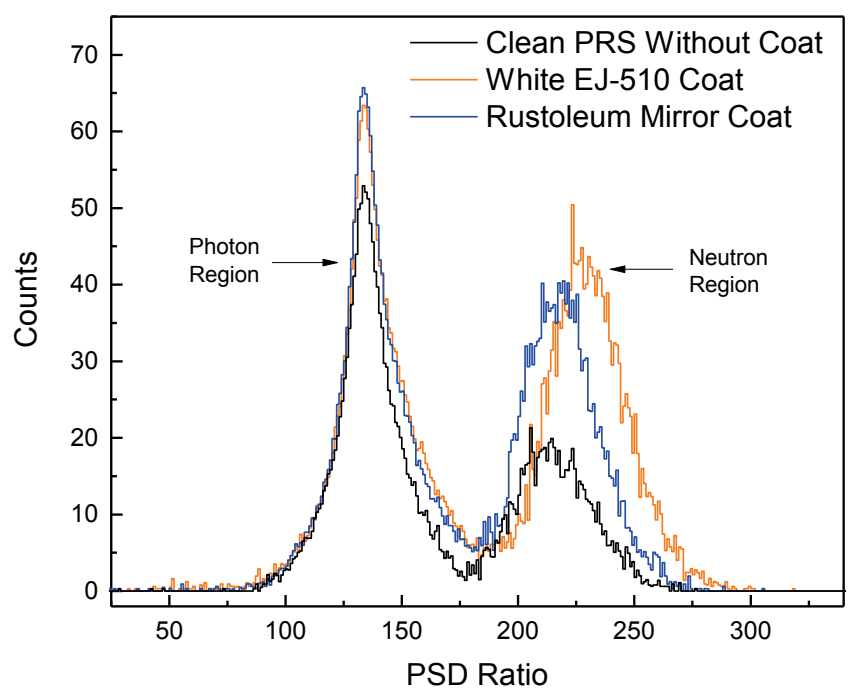

Figure 5. Example of PSD 2-D spectra for three tested PRS coatings. The PSD ratio is tail area/peak area. The FOM applied to these spectra score higher for both coated PRSs for neutron region efficiency and separation of the neutron and photon regions.
TABLE I. COMPARISON OF PSD PERFORMANCE OF PRS BUTTONS WITH THREE DIFFERENT SURFACE TREATMENTS.

\begin{tabular}{ccc} 
Coating & Sensitivity & Figure of Merit \\
\hline Clear (no coating) & $3.5 \pm 0.1$ & 1.42 \\
Rust-Oleum mirror finish & $10.0 \pm 0.1$ & 1.47 \\
EJ-510 white paint finish & $14.3 \pm 0.1$ & 1.66 \\
\hline
\end{tabular}

\section{New PRS Development}

Because the FMMS is a 360 -channel system but only 118 of the 400 reclaimed PRSs were identified for refurbishment, there is need to develop a suitable replacement to ensure all 360 -channels are utilized. Work is now underway to develop a pathway for producing new PRS wafers. Some candidate wafer materials being considered include the following materials.

1. Acrylic cast resin with $5 \% \mathrm{Zns}(\mathrm{Ag})$

2. $\mathrm{PRS}$ reference $\mathrm{ZnS}(\mathrm{Ag})$ (for comparison)

3. Stilbene

4. Acrylic cast resin

5. EJ-34 PSD plastic (painted white in photo)

6. EJ-34A PSD plastic (painted white in photo)

7. EJ-500 optical cement with $5 \% \mathrm{ZnS}(\mathrm{Ag})$

8. EJ-500 optical cement with $20 \% \mathrm{ZnS}(\mathrm{Ag})$

Example buttons made with these materials are shown in Figure 5 (the button labeled as 2 is one of the 118 reclaimed buttons, for comparison). Example scintillation spectra from these wafers are shown in Figure 6.

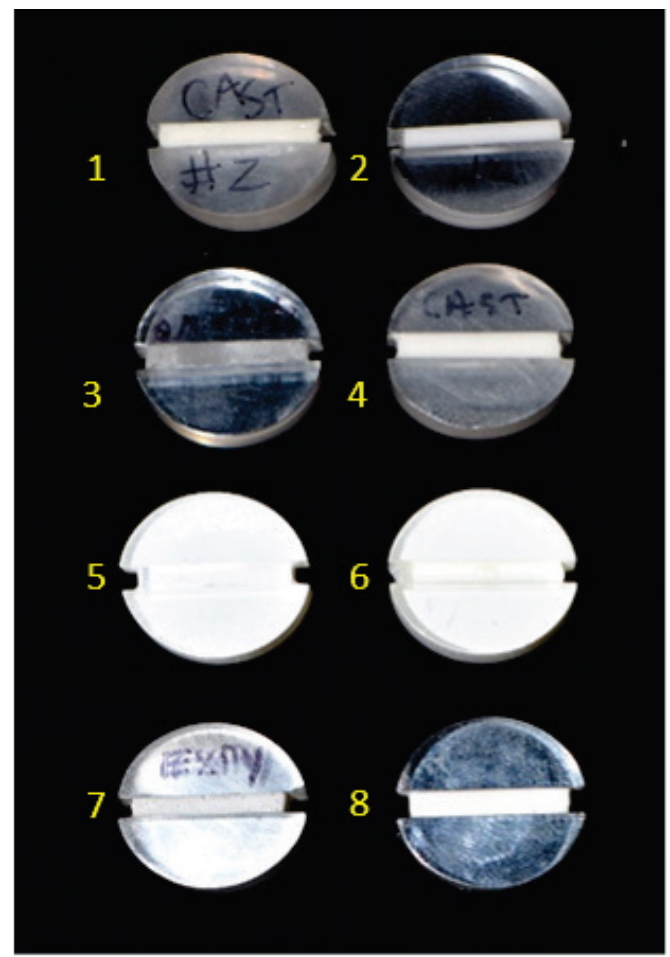

Figure 5. The picture above illustrates candidate PRS wafers. 


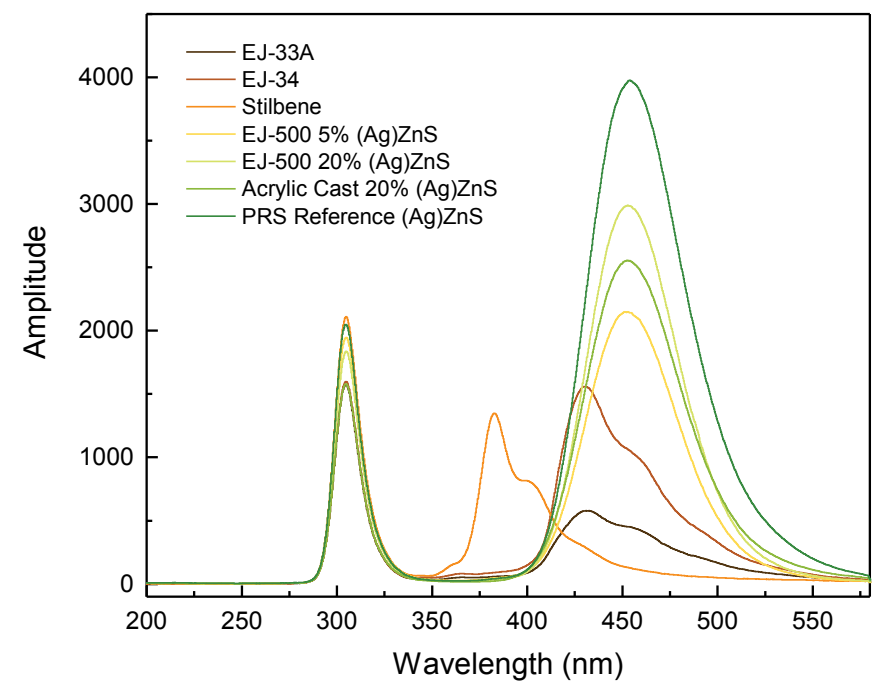

Figure 6. This plot shows a spectrogram with 280 -nm stimulation light, and the output emission light from the candidate PRS wafers.

\section{SUMMARY}

After analyzing all of the PRS detectors, 118 refurbishment candidates were selected out of the 400 reclaimed PRSs. All of the PRS candidates that were selected have white scintillators, no delamination between the scintillators and light guides, and all of the light guides are in alignment. Because the FMMS is a 360-channel array more PRSs are needed and there is a need to develop a suitable PRS replacement. Work is now underway to develop a new pathway for producing PRS wafers.

\section{REFERENCES}

[1] C. Pannier, "The TREAT fast neutron hodoscope: design, refurbishment, and future use ," Idaho National Laboratory report, 18 July 2012.

[2] C.L. Fink, D.L. Smith, R. Daly, "Efficiency measurements on a hornyak button detector and a fission counter using mono-energetic neutrons ," Argonne National Laboratory report April 1977.

[3] Fink, C. L., "Optimization of a Hornyak-Button Detector for FastNeutron Detection," IEEE Trans. Nucl. Sci. NS-29 (1982) 718-721. 